\title{
Investigation of superabsorbent polymer absorbency at reduced chemical potential of water
}

\author{
Ariel Melendres ${ }^{1, *}$, Judy An Antang ${ }^{1}$ and Christian Jake Manacob ${ }^{1}$ \\ ${ }^{1}$ Department of Chemical Engineering, Technological Institute of the Philippines, 363 P. Casal St., Quiapo, Manila \\ 1001, Philippines
}

\begin{abstract}
Different types of superabsorbent polymers (SAP) exhibiting varied absorbency properties were investigated for their performances at varying concentrations of an electrolyte. Water chemical potential, which is a function of concentration and pressure, was interpreted as a major parameter for absorbency and swelling properties of SAP. Superabsorbent polymers that were investigated gave different values of permeability which was carried out in a layer of swelled superabsorbent polymer. This result revealed differences on the structure of the superabsorbent polymers particularly on the crosslinking density. SAP's absorbency properties were further tested and a correlation between the quantity of absorption and the chemical potential of water in terms of sodium chloride concentration was developed using experimental data on free swelling capacity and absorption under pressure. Results showed decreasing quantity of absorption by the superabsorbent polymers with decreasing chemical potential of water with higher reduction in the absorbency at higher pressure applied on the swelled SAP.
\end{abstract}

\section{Introduction}

Superabsorbent polymers are hydrogels of hydrophilic polymer chains that are capable of absorbing and retaining large amount of water and water containing electrolytes [1]. These properties of super absorbent polymer (SAP) are important elements in many of their applications. SAP is composed of repeating units of smaller molecules chemically bound together forming long chains. They are insoluble in any solvent due to the polymer chains being crosslinked by covalent bonds [2-4]. The elastic retractions of the forces of the network prevent the polymer swelling to infinity. Super absorbent polymers that are commercially available in the market are mostly prepared by free radical polymerization [5-6]. What makes the SAP unique is its ability to swallow and retain large amount of water and forms hydrogels. Generally, superabsorbent polymers are lightly crosslinked, partially neutralized hydrophilic three-dimensional networks that expand during water absorption. Their ability to absorb up to several hundred to thousand times of their own dry weight are also influenced by other factors including the $\mathrm{pH}$ of the solution $[7,8]$. Crosslinked polyacrylic acid and polymethacrylic acid, when neutralized with sodium hydroxide, the polymer chains set up an electrostatic repulsion which tends to expand the network, thus providing volumes for diffusion of solvent [9].

Chemical potential is used for describing phenomena and processes. Migration takes place voluntarily, because the chemical potential in the initial state is greater than in the final state [10]. For the swelling of superabsorbent polymer, the solvent located outside of the network, having a higher chemical potential diffuses inside of the network with low chemical potential. At reduced chemical potential, water flow is reduced and eventually stopped when the concentration everywhere is the same. The difference in the concentration of ions between the network of the cross-linked polymer chains and external solvent causes the driving force for the solvent absorption by superabsorbent polymers. The water molecules are then retained inside of the network by the hydrogen bonding. However, it is possible to further limit the flow of the solvent by subjecting the network to a pressure. The chemical potential of the solvent is both a function of the concentration and pressure at constant temperature as shown below.

$$
\mu_{1}=f\left(P, N_{2}\right)
$$

For an ideal solution, the chemical potential is given by the following equation

$$
\mu_{1}=\mu_{1}^{o}+R T \ln \left(1-N_{2}\right)
$$

where $\left(1-N_{2}\right)=N_{1}$ is the mole fraction of solvent in polymer - water solution, $\mu^{\mathrm{o}}{ }_{1}$ is the chemical potential of pure water, $R$ is gas constant and $T$ is temperature. From Equations 1 and 2, the osmotic pressure of the solvent can be derived to give

$$
\pi=\left(\frac{n_{2}}{V}\right) R T
$$

* Corresponding author: arvmelen@yahoo.com 
where $n_{2}$ refers to the number of moles of solute and $V$ is the volume of solution. The osmotic pressure expressed by the above equation refers to the osmotic pressure of the solvent that is located outside of the polymer network. The solvent may either be pure water or mixed with an electrolyte, thus varying the chemical potential of the solvent. The concentration of the electrolyte inside of the polymer network is different from that of the outside creating a concentration gradient. Depending on the chemical potential of water and the pressure applied, the absorption and the swelling capacity of SAP vary at constant temperature.

This paper describes the effect of chemical potential on different types of partially neutralized acrylic based superabsorbent polymer swelling capacity, specifically at reduced chemical potential of water. The effect of chemical potential of water on superabsorbent polymer absorbency was correlated in terms of the free swell absorbency and absorption under pressure. A thorough study on the effect of the chemical potential on surperabsorbent polymers that exhibit varied absorption properties, particularly on the free swelling capacity and absorption under pressure, has not been fully elucidated. This paper describes this research gap, explaining about the correlation of water chemical potential with the free swell capacity (FSC) and absorption under pressure (AUP) of various types of SAP Moreover, it is the objective of this study to provide industry information on on the effect of reduced water chemical potential in the water absorption properties of different SAP, exhibiting variation in polymer structure commonly used in the hygiene industry.

\section{Materials and methods}

\subsection{Materials and reagents}

Three types of commercial superabsorbent polymers (SAP) commonly used for hygienic application were used for the experiments. The SAP were obtained tightly sealed to maintain moisture below 5\%. Analytical grade sodium chloride was obtained from Ajax Finechem. Deionized water with conductivity of $<5 \mu \mathrm{S} / \mathrm{cm}$ was used in preparing sodium chloride solutions.

\subsection{SAP permeability}

$300 \mu \mathrm{m}-600 \mu \mathrm{m}$ of $0.9 \mathrm{~g}$ of dry SAP particles are spread evenly on the 100 mesh stainless steel screen fixed at the bottom of the $6.0-\mathrm{cm}$ diameter acrylic cylinder and allowed to swell for 1 hour in 0.279 mol \% (0.9 \% w) sodium chloride solution producing gel bed of SAP. The permeability is measured with a load of $21 \mathrm{~g} / \mathrm{cm}^{2}$. The weight of 0.279 mol \% sodium chloride passing through the gel bed of SAP is recorded every 5 seconds for 3 minutes and permeability is calculated from the gel height and flow rates.

\subsection{Free swell capacity}

A specified weight of superabsorbent polymer is placed in the heat sealable $70 \mathrm{~mm} \times 70 \mathrm{~mm}$ nonwoven bag. The bag containing the superabsorbent sample is then soaked in the sodium chloride solution for 30 minutes after which the bag is removed. The excess solvent is allowed to drip away, and the bag is weighed. The FSC is evaluated by weighing the bag containing the superabsorbent polymers before and after soaking in the solvent which is calculated as $\mathrm{FSC}=(\mathrm{mw}-\mathrm{ms}-\mathrm{mb}) / \mathrm{ms}$, where $\mathrm{mw}$ is mass of the wet bag containing the sample, $\mathrm{ms}$ is the mass of dry sample and $\mathrm{mb}$ is the mass of the wet blank bag.

\subsection{Absorption under pressure}

SAP particles are spread evenly on the 400 mesh stainless steel screen fixed at the bottom of the $6.0-\mathrm{cm}$ diameter acrylic cylinder. The load of $21 \mathrm{~g} / \mathrm{cm}^{2}$ or $49 \mathrm{~g} / \mathrm{cm}^{2}$ is placed in the cylinder. A porous $10-\mathrm{cm}$ diameter ceramic plate of $5 \mathrm{~mm}$ thickness is placed on the $20-\mathrm{cm}$ diameter petri dish containing the test solution of aqueous sodium chloride. A 7-cm diameter filter paper with pore size of $<25 \mu \mathrm{m}$ is placed on top of the ceramic plate and allow it to wet with the sodium chloride solution. The cylinder apparatus is placed on the center of the wet filter paper to allow SAP absorbs the test solution for 60 minutes. The Absorption Under Pressure (AUP) is calculated from the equation $\mathrm{AUP}=\left(\mathrm{m}_{\mathrm{B}}-\mathrm{m}_{\mathrm{A}}\right) / \mathrm{m}_{\mathrm{s}}$, where $\mathrm{m}_{\mathrm{A}}$ is mass of dry apparatus group before absorption, $\mathrm{m}_{\mathrm{B}}$ is the mass of wet apparatus group after absorption and $\mathrm{m}_{\mathrm{s}}$ is the mass of the sample. All experiments were repeated at least 3 times to make sure of the accuracy of the results. The diagram showing the set-up of the experiment is shown in Figure 1.

\subsection{Experimental set-up}

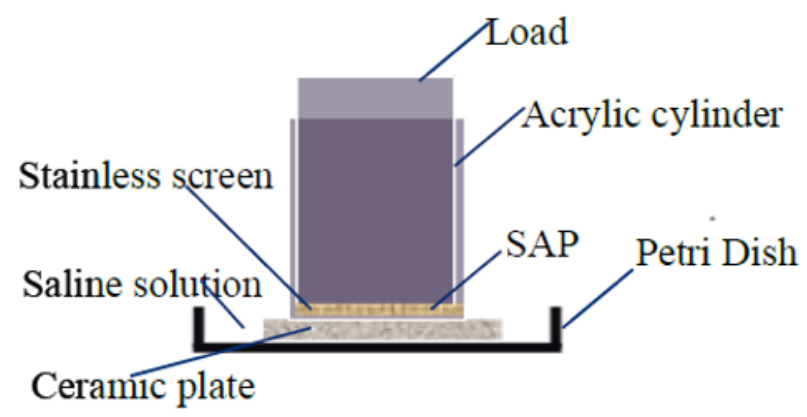

Figure 1. The experimental set-up for Absorption Under Pressure (AUP).

\section{Results and discussion}

The capacity of the superabsorbent to absorb and retain the amount of water depends on the polymer network structure primarily on the crosslink density. It also depends on the degree of neutralization of carboxylic group of starting monomer [7]. It is possible to produce SAP of varied absorption quantities at free swelling condition or under pressure depending on the intended 
application of SAP. Three different types of superabsorbent polymers were tested and the results are shown in Figure 2. The properties shown in Figure 2 are Free Swell Capacity and Absorption Under Pressure at 21 $\mathrm{g} / \mathrm{cm}^{2}$ and $49 \mathrm{~g} / \mathrm{cm}^{2}$ with test liquid of $0.279 \mathrm{~mol} \%(0.9$ $\% \mathrm{w}$ ) of sodium chloride, the test solution normally used for evaluation of SAP by the hygiene industry. Both SAPA and SAP-C have higher Free Swell Capacity, but SAP$\mathrm{B}$ has higher Absorption Under Pressure.

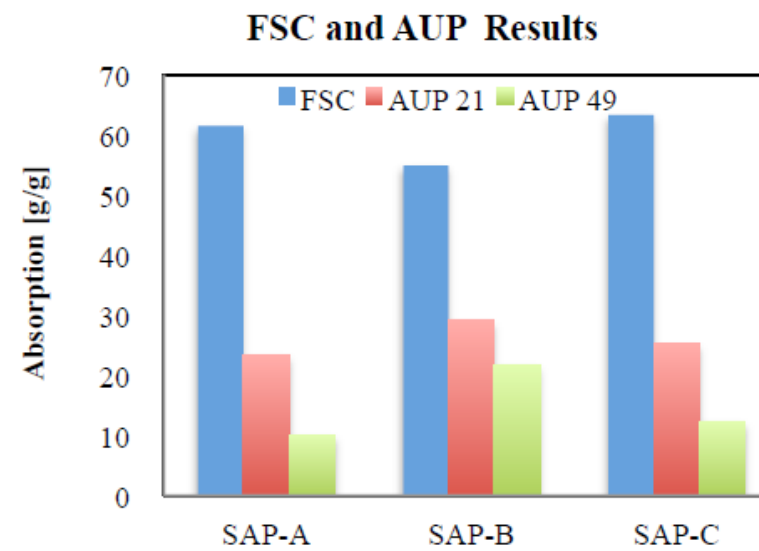

Figure 2. FSC and AUP of SAP-A, SAP-B and SAP-C at $0.279 \mathrm{~mol} \% \mathrm{NaCl}$.

Based on the above results, SAP showed different absorption properties which could be explained in terms of the differences in raw material utilization such as cross linkers, etc., and production process parameters. The properties of the SAP in terms of permeability are shown in Figure 3. SAP-B has the highest permeability value which indicates faster liquid flow through gel layers of SAP-B. The flow rate is shown in the same graph as the secondary y-axis. The permeability as well as the flow rate are mainly affected by the strength of the SAP gel, i.e., the resulting SAP after allowing to swell for 1 hour. The flow rate is used to calculate for the permeability. The gel strength is influenced by the quantity of crosslinkers used in SAP preparation for both inner and surface structure. Hence, results showed three SAP of diverse properties which were apparently had been processed with different crosslinker densities, thus different target applications.

The results of the test for Free Swell Capacity (FSC) using different concentrations of sodium chloride are shown in Figure 4. In the chart, the absorption values of FSC shows decreasing trend with increasing concentration of sodium chloride. The highest quantity of absorption was obtained at pure water. Among sodium chloride water solutions, the highest absorption was obtained at $0.031 \mathrm{~mol} \%$ with lowest at $0.279 \mathrm{~mol} \%$. SAP-C has the highest amount of absorption followed by SAP-A and SAP-B for all the concentrations of sodium chloride used, although SAP-C is only slightly higher than SAP-A with SAP-B much lower than both SAP-C and SAP-A.

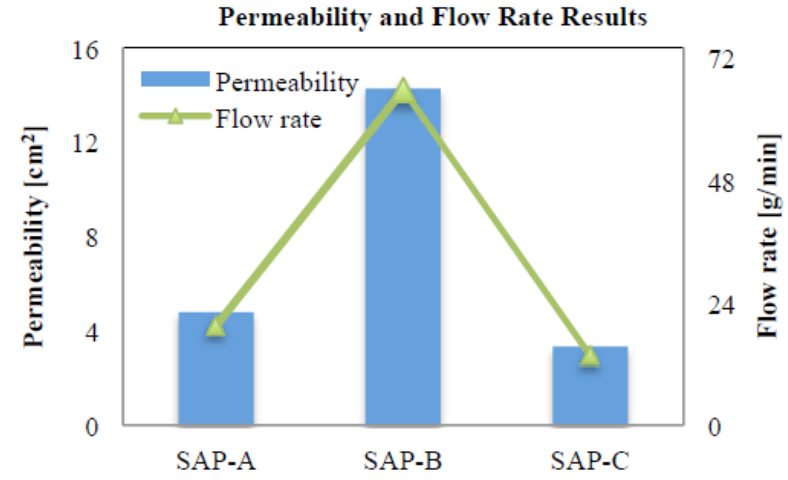

Figure 3. FSC and AUP of SAP-A, SAP-B and SAP-C at $0.279 \mathrm{~mol} \% \mathrm{NaCl}$

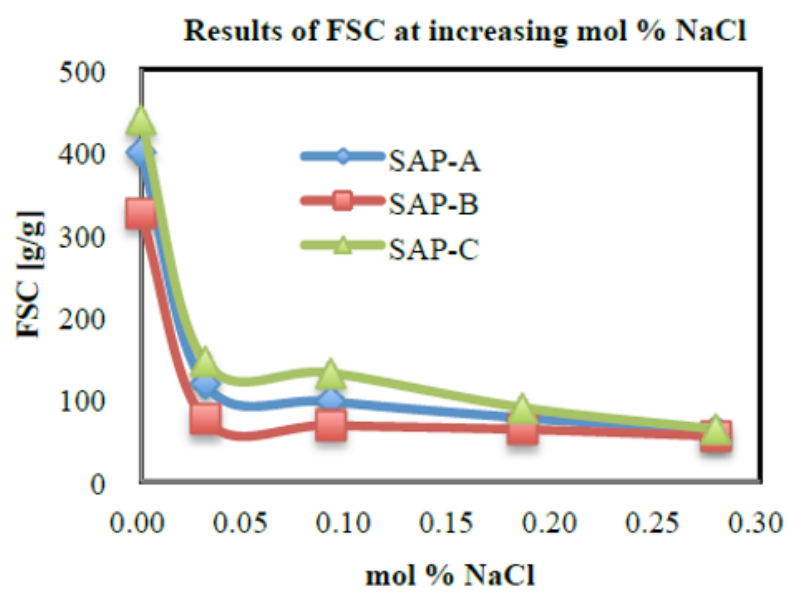

Figure 4. FSC of different SAP at increasing concentration of sodium chloride.

All SAP tested exhibited the same trend of decreasing absorption with the amount of sodium chloride of the test solutions. The result in Figure 4 shows inverse relation of absorption capacity of superabsorbent polymer with the concentration of sodium chloride. Pure water having the highest chemical potential among the test liquids has the greatest ability to flow through the polymer network, compared to that of water-sodium chloride solution. Equation 2 explains this experimental data. The value of the second term in the right side of this equation is always negative, making the value of $\mu_{l}$, the chemical potential of water at impure state, lower than the chemical potential, $\mu^{o}{ }_{1}$, of pure water. Applying Equation 2 for the range of the sodium chloride used in this test, the graph of the change in chemical potential, $\left(\mu_{1}-\mu_{1}^{o}\right)=\Delta \mu_{1}$ is shown in Figure 5. In this graph, $\Delta \mu_{l}$ decreases with increasing concentration of sodium chloride in water and decreasing quantity of absorption as described by the values of FSC for all the SAP tested. 


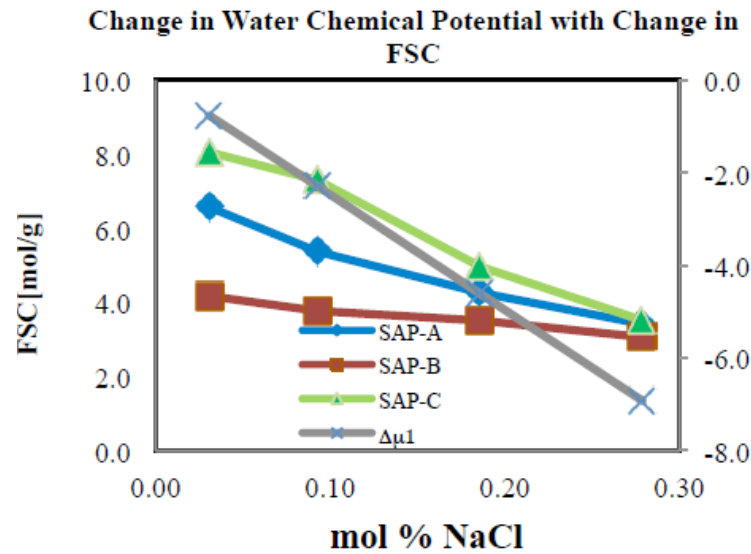

Figure 5. FSC of different SAP with change in water chemical potential

A plot of change in chemical potential shows an inverse relationship with the concentration of sodium chloride. SAP absorbency likewise exhibits inverse relationship with the sodium chloride concentration. The unit of absorbency in Figure 5 is converted in mol per $g$ of SAP. The SAP absorbency, like the chemical potential, decreases with increasing sodium chloride concentration. Different SAP gave diverse degree of response to the change in the chemical potential as their absorbency properties are different based on Figures 2 and 3. For the region where the concentration of the solute sodium chloride in solution ranges from experimental data of 0.031 to 0.279 mole percent, the change in absorption quantity per mole of water decreases with reduction of chemical potential. Figure 6 and Figure 7 show the results of test done for absorption under pressure at $21 \mathrm{~g} / \mathrm{cm}^{2}$ and $49 \mathrm{~g} / \mathrm{cm}^{2}$ respectively. In this test, the superabsorbent polymers were allowed to absorb the test solutions under load placed directly onto the superabsorbent polymers. In Figure 6 , the value of absorption under pressure shows decreasing trend with the concentration of sodium chloride for all the SAP tested. Although all SAP exhibited the same trend, the values of AUP vary with the type of SAP.

Generally, all SAP tested gave lowest value of AUP at $0.279 \mathrm{~mol} \%$ wherein SAP-A gave the lowest value of AUP for all the concentrations tested compared to other two other SAP's. SAP-C gave higher value of AUP except at $0.279 \mathrm{~mol} \%$ where SAP-B absorbs more test liquid. Figure 7 shows the value of absorption under pressure at higher load of $49 \mathrm{~g} / \mathrm{cm}^{2}$. SAP-B gave much higher values of AUP than SAP-A and SAP-C. This separates the property of SAP-B with that of SAP-A and SAP-C in terms of AUP which shows that SAP-B is a high AUP superabsorbent polymer.

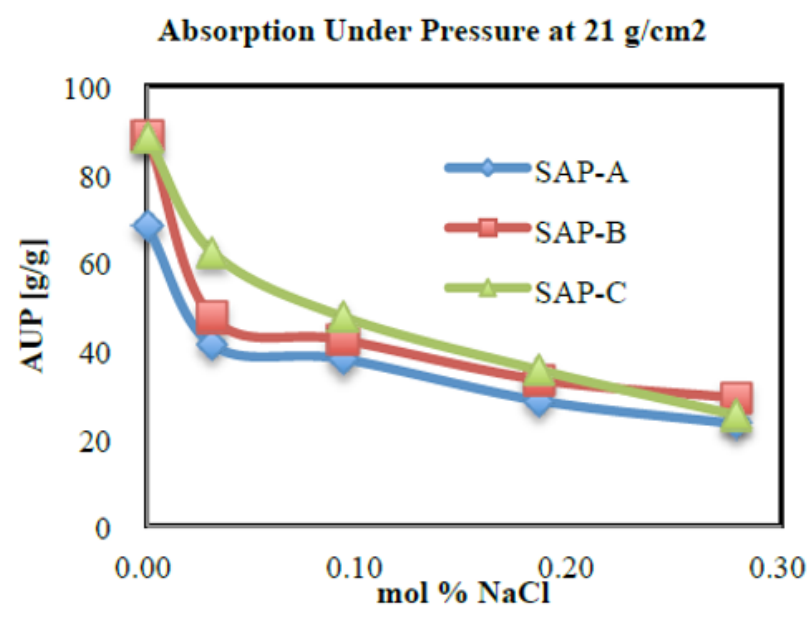

Figure 6. AUP at $21 \mathrm{~g} / \mathrm{cm}^{2}$ of different SAP at increasing concentration of sodium chloride

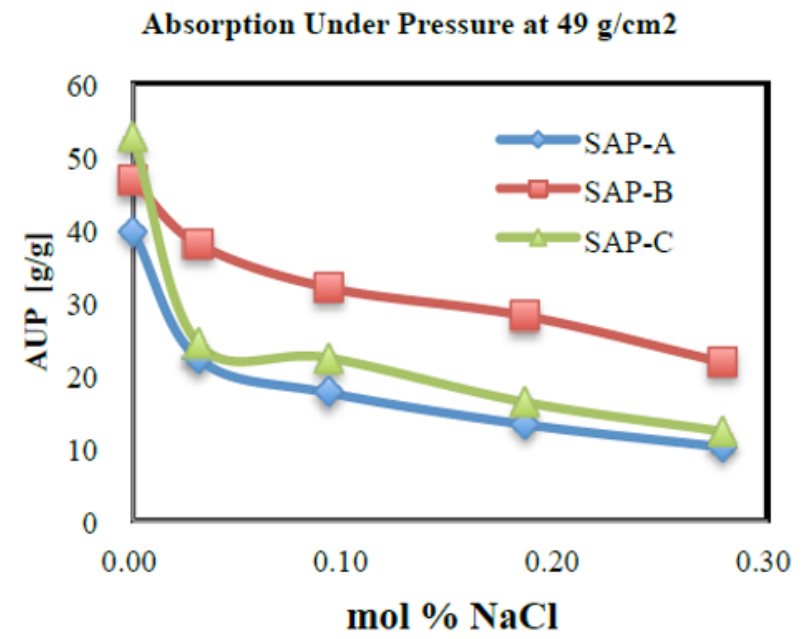

Figure 7. AUP at $49 \mathrm{~g} / \mathrm{cm}^{2}$ of different SAP at increasing concentration of sodium chloride

The property of SAP-B of having high AUP can be seen as a consequence of having high permeability but lower FSC as shown in Figures 2 and 3 compared with SAP-A and SAP-C. The results in Figure 6 and 7 can be generalized as that every SAP tested gave decreasing absorption under load with increasing pressure. SAP absorption capacity decreased when it is allowed to absorb the aqueous solution under pressure. Recall that the three-dimensional network of the polymer chains expands when mixed with water creating a volume for the water to come in to fill the network. The volume created depends on the crosslinking density and the elasticity of the polymer chains. At the elastic limit of the polymer network, the maximum amount of the water fills the entire network. According to Equation 2, for the range of sodium chloride used in the experiment, the decrease in absorption is proportional to the chemical potential thus,

$$
Q=-k \ln \left(1-N_{2}\right)+C
$$

where $Q$ is the quantity of absorption in grams of water solution absorbed per gram SAP for corresponding solute mole fraction, $N_{2}$, obtained from the experimental values 
of FSC and AUP. The values of $k$ and $C$ for SAP-A is evaluated using Equation 4 and listed in Table 1 where AUP21 and AUP49 refer to the absorption under pressure at $21 \mathrm{~g} / \mathrm{cm}^{2}$ and $49 \mathrm{~g} / \mathrm{cm}^{2}$ respectively.

Table 1. Values of constants for SAP-A

\begin{tabular}{lccc}
\hline Constant & FSC & AUP21 & AUP49 \\
\hline$k$ & -211.92 & -59.61 & -45.43 \\
$C$ & 123.90 & 42.55 & 23.28 \\
\hline
\end{tabular}

As shown in Table 1, the values of $k$ for FSC data are lower than that of AUP. The values of $C$ gave an opposite relation. The same trends were also observed for SAP-B and SAP-C. The right side, first term of Equation 4 represents the negative effect in the swelling of SAP, which increases negatively as the concentration of sodium ions increased as a result of the weakening of the chemical potential of water. The second term, constant $C$, corresponds to the positive effect on the swelling which is independent of the concentration of the sodium chloride used for a particular $k$ value.

\section{Conclusion}

The chemical potential of water is an important factor that determines the swelling capacity of superabsorbent polymers. Chemical potential of pure water decreases with increasing amount of solute, thus decreasing the ability to flow through the network of the superabsorbent polymer. SAP of different level of absorption capacities, having different gel bed permeabilities, exhibited the same trend of decreasing absorption capacities with the decrease of chemical potential. A correlation between the quantity of absorption with the concentration of solute in water mixture was developed for a range of solute concentration. It was also demonstrated that the quantity of absorption is influenced by load on the polymer network at different electrolyte concentrations.

This research was under the Balik Scientist Program of Department of Science and Technology with the host institution Technological Institute of the Philippines.

\section{References}

[1] Ahmed,E. M., Hydrogel: Preparation, Characterization And Applications: A Review. J.Adv. Res.,6, 2015, pp. 105-121

[2] Matanovic M. R., Kristl, J. Grabnar P. A. Thermoresponsive polymers: Insights into decisive hydrogel characteristics, mechanisms of gelation, and promising biomedical applications. Int. J. Pharm. 472, 2014, 262-275

[3] Peppas N. A., Mongia, N. K. Ultrapure poly(vinyl alcohol) hydrogels with mucoadhesive drug delivery characteristics. Eur. J. Pharm. Biopharm. 43, 1997, 51-58.

[4] Cheng, D., Liu, Y., Yang, G., Hao G., Wang, Y., Zhang, A. Preparation of low cost superabsorbent hydrogel by urea and acrylic acid. Materials Letters. 204, 2014, 16-18.

[5] Zhang, J., Xiao, H., Li, N., Ping, Q., Zhang, Yi. Synthesis and characterization of superabsorbent hydrogels based on hemicellulose. Journal of applied polymer. 132, 2015,34

[6] Omidian, H., Hashemi, S. A., Sammes, P. G., Meldrum, G. Modified acrylic-based superabsorbent polymers.Polymer. 39, 1998, 3459-3466

[7] Adair, A., Klinpituksa, P., Kaesaman, A. Influences of neutralization of superabsorbent hydrogelfrom hydroxyethyl cellulose on water swelling capacities. The $4^{\text {th }}$ Conference on research, implementation and education of mathematics and science. 1868(1), 2017, 10.1063/1.4995098

[8] Tanaka, T. and Fillmore, D. J. Journal of Physical Chemistry. 70, 1979, 1214

[9] Flory P J: Principles of Polymer Chemistry. Cornell University Press, 1953, 580-585

[10] Job, G., Hermann, F. Chemical Potential- a quantity in search of recognition. European Journal of Physics, 27, 2006, 353-371 